2. Verhoye JP, de Latour B, Heautot JF, Langanay $\mathrm{T}$, Corbineau H, Leguerrier A, et al. Mid-term results of endovascular treatment for descending thoracic aorta disease in high-surgical risk patients. Ann Vasc Surg. 2006;20:714-22.

3. Gawenda M, Aleksic M, Heckenkamp J, Reichert V, Gossmann A, Brunkwall J. Hybrid-procedures for the treatment of thoracoabdominal aortic aneurysms and dissections. Eur J Vasc Endovasc Surg. 2007;33:71-7.
4. Mohammadi S, Voisine P, Mathieu P, Dagenais F. Use of the endoclamp device in a patient with a native mitral valve endocarditis and a previous arch replacement with a modified Griepp technique. J Thorac Cardiovasc Surg. 2006;131:1188-90.

5. Anwar S, Al-Khattab Y, Williams GT. An operative method for surgical revision of a late failure after endovascular repair of an abdominal aortic aneurysm. J Vasc Surg. 2001;34:357-9.

\title{
Postural change causing leg malperfusion resulting from expansion of a patent false lumen in type $B$ aortic dissection
}

\author{
Atsushi Nakahira, MD, ${ }^{\mathrm{a}, \mathrm{b}}$ Hitoshi Ogino, MD, ${ }^{\mathrm{a}}$ Hitoshi Matsuda, MD, ${ }^{\mathrm{a}}$ \\ Kenji Minatoya, MD, ${ }^{a}$ Hiroaki Sasaki, MD, Junjiro Kobayashi, MD, ${ }^{a}$ \\ Toshikatsu Yagihara, MD, and Soichiro Kitamura, MD, ${ }^{a}$ Osaka, Japan
}

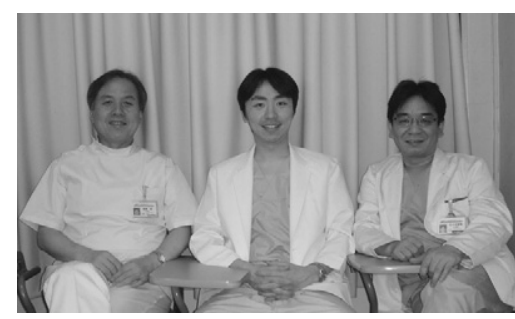

Drs Ogino, Nakahira, and Sasaki (left to right)

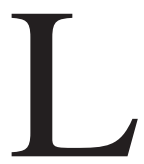

eg malperfusion is an important symptom resulting from aortic dissection (AoD). ${ }^{1}$ Type B AoD, especially with a patent false lumen, exhibits various and complex clinical manifestations. There are no previous reports regarding leg malperfusion caused by postural change. We report 2 interesting cases of patients with type B AoD and a patent false lumen who presented with intermittent claudication despite an ankle brachial pressure index (ABPI) of 1.0.

\section{Clinical Summary}

Case 1. A computed tomographic (CT) scan in a 73-year-old man with acute type B AoD revealed that the patent false lumen was tense. Several months later, the patient was examined again with intermittent claudication, but a follow-up CT revealed no specific change. When the patient was supine, the ABPI was 1.0 in both legs and duplex ultrasound of the popliteal artery showed a normal flow pattern (Figure 1, C). However, the reverse phase flow disappeared and the diastolic phase flow increased when the patient stood up (Figure 1,D). We suspected that the true lumen was further compressed to a paper-thin sliver when the patient stood up (Figure 1, $A, B$ ), and intermittent claudication occurred. An axillo-bifemoral bypass was electively performed for extra-anatomic revascularization. Postoperatively, the duplex ultrasound revealed a normal

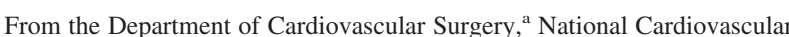
Center, Osaka, Japan; and Department of Cardiovascular Surgery, ${ }^{\mathrm{b}}$ Osaka City University Graduate School of Medicine, Osaka, Japan.

Received for publication May 1, 2007; accepted for publication May 30, 2007.

Address for reprints: Hitoshi Ogino, MD, Department of Cardiovascular Surgery, National Cardiovascular Center, 5-7-1, Fujishirodai, Suita, Osaka, 565-8565, Japan (E-mail: hogino@hsp.ncvc.go.jp).

J Thorac Cardiovasc Surg 2007;134:1046-7

$0022-5223 / \$ 32.00$

Copyright @ 2007 by The American Association for Thoracic Surgery doi:10.1016/j.jtcvs.2007.05.047 flow pattern regardless of postural change (Figure 1, E, F), and the symptom resolved.

Case 2. Intermittent claudication with numbness of the left leg developed in a 47-year-old man with acute type B AoD. CT revealed that the false lumen was patent and that a dissection flap extended to the right common iliac artery, where there was a small reentry tear (Figure 2, A). Although the ABPI was 1.0 in both legs when the patient was supine, his left leg lost pulsation and the blood pressure diminished with a symptom of numbness after walking. It appeared that the true lumen was compressed by further expansion of the patent false lumen resulting from the postural change and walking exercise, and that the bloodstream of his left leg was severely disturbed (Figure 2, $B, C)$. Surgical aortic fenestration above the abdominal aortic bifurcation (Figure 2,D) was performed, and the symptom resolved.

\section{Discussion}

The treatment strategy for type B AoD is still controversial and is made on an individual basis. ${ }^{2}$ Even in patients who survive until hospital discharge, the mortality is insufficient, despite improved surgical techniques and the advent of new endovascular therapies, such as stenting and fenestration. ${ }^{2}$ Akutsu and colleagues ${ }^{3}$ demonstrated that false lumen patency is a strong independent prognostic factor for type B AoD. The anatomic position of the intimal flap is important with regard to peripheral malperfusion of branch vessels. Gaxotte and colleagues ${ }^{4}$ reported that evaluation of the intimal flap position was important and useful for endovascular treatment strategies.

In our 2 cases, the symptom of intermittent claudication had some discrepancies from the ABPI. Although the precise mechanism of this unique manifestation is difficult to prove, we propose that postural change is a key factor in the atypical leg malperfusion. A possible mechanism is that the true lumen is compressed by further expansion of the patent false lumen, such that the bloodstream in the true lumen diminishes when a patient changes his or her position to standing. A duplex ultrasound study of the popliteal artery in the standing position in case 1 and an exercise test in case 2 were effective for elucidating the unique mechanisms of the atypical leg malperfusion. After surgi- 


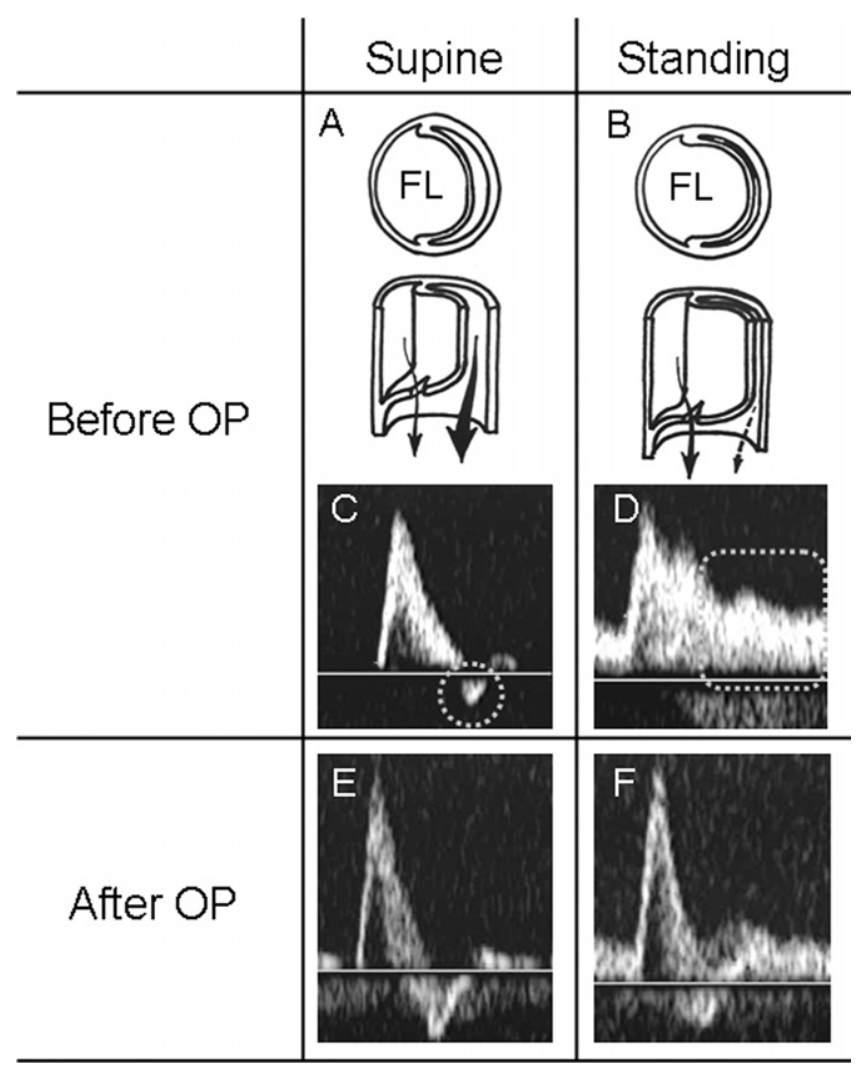

Figure 1. Case 1. A and B, Schemas of the intimal flap position depending on the patient's position show that the patent false lumen expands further when the patient stands up. C-F, Duplex ultrasound scanning of the popliteal arterial flow reveals a loss of reverse phase flow (C, gray dotted circle) and an increase in diastolic phase flow ( $D$, gray dotted rectangle) when the patient stands up. After revascularization through an extra-anatomic bypass, the reverse phase flow of the popliteal artery does not change, even when the patient stands up ( $E$ and $F$ ). FL, False lumen; $\boldsymbol{O P}$, operation.

cal revascularization, the symptom resolved in both cases with a continuous bloodstream to the lower legs.

Panneton and colleagues ${ }^{5}$ reported that surgical aortic fenestration for chronic AoD had durable benefits for carefully selected patients, and the patient in case 2 underwent surgical aortic fenestration. Percutaneous endovascular fenestration was recently reported to be useful for treating acute $\mathrm{AoD}^{4}$ and may represent an alterative intervention in our cases.

Limb ischemia secondary to AoD is predictive of death and visceral ischemia, ${ }^{1}$ and the atypical leg malperfusion in our cases may be a sign of subclinical visceral malperfusions. If patients with type $\mathrm{B} A \mathrm{AD}$ and a patent false lumen have intermittent claudication with a normal ABPI, we should carry out examina-

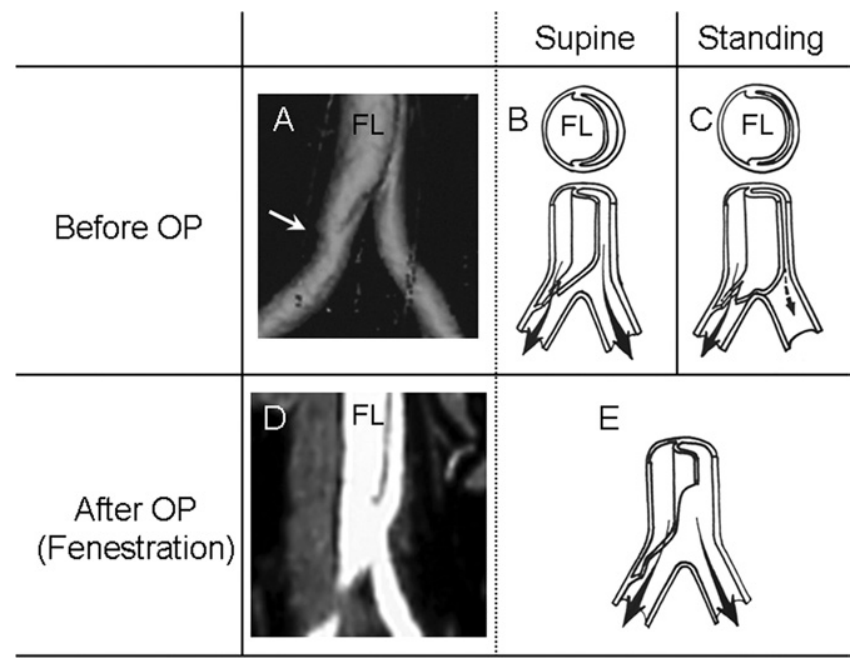

Figure 2. Case 2. A, Three-dimensional reconstructed CT scan demonstrates an intimal flap just above the bifurcation of the abdominal aorta. B and C, Schemas of the intimal flap position depending on the patient's position show that the patent false lumen expands further and that the true lumen becomes collapsed (C) when the patient stands up. D and $E$, Coronal view of an enhanced CT (D) and schema (E) of the aortic bifurcation after revascularization by surgical aortic fenestration reveal good communication between the true and false lumens. $F L$, False lumen; $O P$, operation.

tions that can be performed in both the supine and the standing positions, considering that postural change may cause leg malperfusion. Radiologic examinations and the ABPI provide information for only the supine position. In conclusion, patients with type B AoD and a patent false lumen should be carefully followed, considering the postural reversibility of branch malperfusions.

\section{References}

1. Henke PK, Williams DM, Upchurch GR, Proctor M, Cooper J, Fang J, et al. Acute limb ischemia associated with type B aortic dissection; clinical relevance and therapy. Surgery. 2006;140:532-40.

2. Tsai TT, Fattori R, Trimarchi S, Isselbacher E, Myrmel T, Evangelista A, et al. Long-term survival in patients presenting with type B acute aortic dissection. Circulation. 2006;114:2226-31.

3. Akutsu K, Nejima J, Kiuchi K, Sasaki K, Ochi M, Tanaka K, et al. Effects of the patent false lumen on the long-term outcome of type B acute aortic dissection. Eur J Cardiothorac Surg. 2004;26: 359-66.

4. Gaxotte V, Cocheteux B, Haulon S, Vincentelli A, Lions C, Koussa M, et al. Relationship of intimal flap position to endovascular treatment of malperfusion syndromes in aortic dissection. $J$ Endovasc Ther. 2003; 10:719-27.

5. Panneton JM, Teh SH, Cherry KJ Jr, Hofer JM, Gloviczki P, Andrews JC, et al. Aortic fenestration for acute or chronic aortic dissection: an uncommon but effective procedure. J Vasc Surg. 2000;32:711-21 